\title{
La universidad pública y la formacion de talento humano ante la pandemia por la COVID-19
}

\section{The public university and the training of human talent in the face of the COVID 19 pandemic}

NUÑEZ, Martha L. ${ }^{1}$

HENAO, Angela M. ${ }^{2}$

FAJARDO, Elizabeth ${ }^{3}$

\begin{abstract}
Resumen
El objetivo del trabajo fue sintetizar la evidencia relacionada con la necesidad del talento humano cualificado en enfermería en el cuidado de personas en condición de salud crítica. Se utilizó como metodología el Rapid Review, según la OMS. Sedetermino que en Colombia se ofertan 5 programas de especialización en enfermeira en cuidado critico, lo que llevó a concluir que durante la pandemia por COVID-19 el personal de enfermeria calificado, con especializacion en cuidado de personas en condicion de salud critico, es limitado, lo que lleva a las instituciones de educaciòn a responder ante la formacion de este recurso humano haciendo frente a las problemáticas de salud de la comunidad y su entorno.

Palabras clave: especialización, educacion superior, cuidados criticos
\end{abstract}

\begin{abstract}
The objective of the work was to synthesize the evidence related to the need for qualified human talent in nursing in the care of people in critical health conditions. The Rapid Review was used as the methodology, according to the WHO. It was determined that in Colombia 5 specialization programs in critical care nursing are offered, which led to the conclusion that during the COVID-19 pandemic, qualified nursing personnel, specializing in the care of people in critical health conditions, is limited, which leads educational institutions to respond to the training of this human resource by facing the health problems of the community and its environment.
\end{abstract}

key words: specialization, higher education, critical car

\section{Introducción}

La Ley 30 (Congreso de la República de Colombia, 1992), por medio de la cual se organizó el sistema de Educación Superior en Colombia, está a punto de cumplir 30 años y han sido diferentes las acciones que el Estado a través del Ministerio de Educación Nacional ha emprendido con el fin de consolidar el sistema de Educación Superior con el propósito de dar respuesta a las necesidades cambiantes del contexto.

Dicha ley, en su capítulo $V$ artículo 16, define la tipología de Instituciones de Educación Superior (IES) describiendo tres tipos; en cuya composición define como tercer eslabón a las universidades otorgándoles como

\footnotetext{
${ }^{1}$ Profesora Asociada. Facultad de Ciencias de la Salud . Universidad del Tolima. https://orcid.org/0000-0002-2012-6554

2 Profesora asistente. Facultad de Enfermeria. Universidad Nacional de Colombia. Orcid. https://orcid.org/0000-0003-4203-0016

${ }^{3}$ Profesora Titular. Facultad de Ciencias de la Salud . Universidad del Tolima. Orcid. https://orcid.org/0000-0002-3484-1620
} 
función la formación de profesionales, el desarrollo y divulgación del conocimiento y generación desde la académia de fuerza laboral con niveles educativos que permitan realizar impactos sobre el entorno o en otros términos una relación articulada y productiva en la simbiosis universidad-sociedad.

De igual manera, se ha planteado desde la normativa, el objetivo fundamental de garantizar a la sociedad que las instituciones que hacen parte del sistema cumplen los más altos requisitos de calidad y que materializan los propósitos y objetivos planteados en los documentos que los acreditan como IES (Gómez \& Celis, 2009).

Desde finales de la década de los 90, los términos calidad, registro calificado y acreditación con todas sus implicaciones, han tomado especial relevancia en las directrices impartidas por parte del MEN, llevando a que las IES comenzaran a planear su oferta académica, a organizar sus procesos de formación, investigativos, de proyección social y administrativos con el fin de poder responder a los altos estándares de calidad que a través de los diferentes lineamientos y procedimientos que se han venido instaurando para las IES.

Este artículo tiene como objetivo sintetizar la evidencia relacionada con la necesidad del talento humano cualificado en enfermeria en el cuidado de personas en condición de salud crítica. Es en este contexto en el que se mueve la Universidad del Tolima, una universidad pública colombiana, de orden regional; que superando las fronteras del Departamento del Tolima, hoy hace presencia en las regiones mas apartadas del país y cuenta con mas de 21.000 estudiantes matriculados. En julio de 2020, obtuvo su acreditación institucional de alta calidad y uno de sus grandes logros es contar con una Facultad de Ciencias de la Salud con sus dos programas (Enfermería y Medicina), acreditados en alta calidad por el Consejo Nacional de Acreditación (CNA) y el programa de Enfermeria con acreditación internacional ARCUSUR (Sistema de Acreditacion de Carreras Universitarias) (Universidad del Tolima, 2018).

Estos reconocimientos cobran vital importancia en razón a que, como institución formadora de talento humano en salud tanto en el nivel de pregrado como de posgrados, pone de presente su rol como actor fundamental en la propuesta de respuestas acertadas a las necesidades que plantea la pandemia por la COVID 19 e instaurar soluciones efectivas a los problemas de salud de las comunidades sobre las que hace cobertura.

La infección por coronavirus causante de la pandemia por COVID-19, genera cuadros clínicos con diferentes niveles de gravedad, evolucionando en los casos mas graves a síndromes de dificultad respiratoria de niveles variables que precisan el traslado a unidades de cuidado intensivo y soporte mecánico ventilatorio (Chica, y otros, 2020). La experiencia en el cuidado de personas en condición de salud crítica, ha enseñado que la recuperación de individuos en tales condiciones, se logra cuando se cuenta con herramientas tecnológicas de punta para el diagnóstico y tratamiento, suministro oportuno de medicamentos y lo mas importante el talento humano cualificado para la utilización correcta de las herramientas disponibles y la implementación de los cuidados requeridos segundo a segundo.

La medicina crítica y el cuidado intensivo, como área de estudio a nivel posgradual en todas las profesiones que involucran el cuidado de la salud humana, apareció de manera relativamente reciente en el desarrollo de esta área del conocimiento. La creación de salas de cuidado especializado en el cuidado de personas en condiciones criticas de salud y en riesgo de morir, con todos los criterios de cuidado especializado, aparecieron en la década de los 50 y 60 en Estados Unidos y Europa, y a mediados de 1969 en Colombia. En sus inicios, la medicina crítica y el cuidado intensivo eran dirigidos y manejados por cirujanos y médicos dedicados a cuidar los posoperatorios de cirugías mayores o a enfermos con insuficiencia respiratoria que requerían soporte ventilatorio (Matiz, 2016), siendo prácticamente una extensión de la unidad de recuperación quirúrgica, muchas veces solo bajo el cuidado y la responsabilidad de personal de enfermería y terapeutas sin entrenamiento.

Las condiciones de calidad requeridas para el funcionamiento de las unidades de cuidado intensivo se encuentran reglamentadas no solamente por los acuerdos establecidos por las asociaciones académicas en cada 
especialidad, sino por la normativa en salud de cada país. En Colombia el sistema único de habilitación "Es el conjunto de normas, requisitos y procedimientos mediante los cuales se establece, registra, verifica y controla el cumplimiento de las condiciones básicas de capacidad tecnológica y científica, de suficiencia patrimonial y financiera y de capacidad técnico administrativa, indispensables para la entrada y permanencia en el Sistema, los cuales buscan dar seguridad a los usuarios frente a los potenciales riesgos asociados a la prestación de servicios y son de obligatorio cumplimiento por parte de los prestadores de servicios de salud y las empresas administradoras de planes de beneficios" (Ministerio de Salud y Protección Social, 2016) y se encuentra reglamentado mediante el decreto 780 de 2016, que establece el nivel de formación de médicos y enfermeras en los servicios de cuidado crítico entre otros.

La Universidad del Tolima, realiza cobertura no solamente en procesos de formación sino en la propuesta de soluciones al municipio de Ibagué (capital del Departamento del Tolima) ubicado en el centro de Colombia. Con motivo de la pandemia son múltiples las oportunidades de intervención desde la única IES pública del Departamento. Teniendo en cuenta su potencial como IES líder en la formación de talento humano en salud, es pertinente abordar la pregunta: ¿cuál es la necesidad de talento humano cualificado de enfermeras expertas en el cuidado de personas en condición de salud crítica en el Municipio de Ibagué?

\section{Metodología}

Se llevó a cabo una revisión tipo Rapid Review como una estrategia de generación de conocimiento, permitiendo sintetizar los hallazgos y evaluar la validez de la evidencia de la investigación utilizando Métodos de revisión sistemática "abreviados", modificando estos métodos para generar evidencia en poco tiempo. (Tricco, Antony, Zarin, Strifler, Ghassemi, Ivory, Perrier, Hutton, Moher \& Straus. 2015) (Khangura, Konnyu, Cushman, Grimshaw \& Mohe. 2012). Especialmente de informacion ya conocida de la cual se busca extraer los aportes que contribuyan a identificar la pregunta de interés (Grant \& Booth, 2009), en este caso, ¿cuál es la necesidad de talento humano cualificado de enfermeras expertas en el cuidado de personas en condición de salud crítica en el Municipio de Ibagué?. La búsqueda se realizó en documentos de texto completo, especialmente revisión de paginas web de IES nacionales e internacionales, bases de datos del SNIES, del sistema de vigilancia SIVIGILA, revisión de las paginas web de universidades colombianas y extranjeras en las cuales se oferta la educación continua de cuidado crítico adulto permitiendo generar tres categorías.

Se incluyeron artículos e informes relacionados con la pregunta, se evaluaron y compararon de manera independiente por cada uno de los investigadores. Los criterios de selección se establecieron relacionando el papel de la educacion en cuidado crítico y el impacto de la fuerza laboral en la pandemia. Después de que se observó un acuerdo> 90\% entre los evaluadores, se analizaron los resultados de la búsqueda en la literatura de forma independiente y las discrepancias se resolvieron mediante discusión. Toda la informaciòn se recopilo en la plantilla desarrollada por los investigadores para tal fin.

\section{Resultados}

Después de realizar las revisiones planteadas en la metodología, se encontraron tres categorías de respuestas que sustentan el rol de la Universidad del Tolima como formadora de talento humano en salud para dar respuesta a la pandemia y que se desarollan a continuación: la primera relacionada con los programas de especialidad en enfermería en cuidado crítico disponibles en Latinoamérica y en Colombia (las oportunidades de formación para graduados de enfermería); la segunda relacionada con la disponibidad de profesionales cualificados para atender la pandemia en Colombia; y la tercera, el contexto de número de camas disponibles para la atención de pacientes con Covid en el Tolima y los profesionales cualificados disponibles. 


\subsection{Programas de formación a nivel internacional y en Colombia. Oportunidades de formación para la atención de pacientes con Covid.}

Enfermería como disciplina científica abocada al cuidado de las personas se ve comprometida con el desarrollo de competencias específicas para los ambientes de cuidado, tal es el caso del Cuidado Intensivo, donde realiza su práctica diaria. Los Cuidados Intensivos de Enfermería han evolucionado a lo largo de los años, el personal de enfermería que presta servicio en la Unidad de Cuidados Intensivos (UCI) debe tener una formación específica en competencias de cuidados críticos que le aporte un profundo conocimiento científico de los procesos fisiopatológicos y de las respuestas del paciente a la enfermedad (Gonzalez, 2015).

En la tabla numero 1 pueden evidenciarse algunos programas latinoamericanos que ofertan programas de especialización en Enfermería, a los que podrían acceder profesionales de Enfermería Colombianos.

Tabla 1

Programas de especialización para

enfermeras en el área de cuidado crítico.

\begin{tabular}{|c|c|c|c|c|}
\hline País & Denominación & Institución & Duración & Objetivo \\
\hline Argentina & $\begin{array}{l}\text { Especialización en } \\
\text { Enfermería en la Atención } \\
\text { del Paciente Crítico Adulto }\end{array}$ & $\begin{array}{l}\text { Universidad } \\
\text { Nacional del Tres } \\
\text { de Febrero }\end{array}$ & 1 año & $\begin{array}{l}\text { Satisfacer la necesidad del sistema salud de contar } \\
\text { con enfermeros especializados en la atención de } \\
\text { adultos críticamente enfermos. Para ello, se ofrece } \\
\text { un programa diseñado para la formación de } \\
\text { profesionales reflexivos, con pensamiento crítico y } \\
\text { capacitado para la toma de decisiones con sustento } \\
\text { en fundamentos científicos en todas las funciones de } \\
\text { enfermería }\end{array}$ \\
\hline Argentina & $\begin{array}{l}\text { Especialización en } \\
\text { Enfermería en la Atención } \\
\text { del Paciente Crítico Adulto }\end{array}$ & $\begin{array}{c}\text { Universidad } \\
\text { Favaloro }\end{array}$ & 2 años & $\begin{array}{l}\text { Ofrecer a los estudiantes la posibilidad de formarse } \\
\text { en la atención del paciente crítico adulto, } \\
\text { enriqueciendo su desarrollo personal, técnico y } \\
\text { científico. Formar un especialista con un perfil } \\
\text { claramente humanístico y sólida formación } \\
\text { profesional, científica y técnica. Perfeccionar al } \\
\text { estudiante en la utilización del método científico } \\
\text { como instrumento útil para la solución de problemas. }\end{array}$ \\
\hline Chile & $\begin{array}{l}\text { Programa de } \\
\text { Especialización de } \\
\text { Enfermería en Cuidados } \\
\text { del Paciente Crítico Adulto }\end{array}$ & Andes & 2 años & $\begin{array}{l}\text { Desarrollar competencias teórico-clínicas en la } \\
\text { entrega de cuidados de enfermería fundamentados, } \\
\text { oportunos y seguros, otorgados al paciente } \\
\text { críticamente enfermo con uso de la práctica basada } \\
\text { en evidencia que fomente el pensamiento crítico y la } \\
\text { toma de decisiones del enfermero que se } \\
\text { desenvuelve en el ámbito clínico. }\end{array}$ \\
\hline México & $\begin{array}{l}\text { Especialización de } \\
\text { Enfermería en Cuidados } \\
\text { Intensivos del Adulto en } \\
\text { Estado Crítico }\end{array}$ & $\begin{array}{l}\text { Universidad } \\
\text { Veracruzana }\end{array}$ & 3 semestres & $\begin{array}{l}\text { Formar Especialistas en Cuidados Intensivos en el } \\
\text { Adulto en Estado Crítico, altamente calificados en la } \\
\text { gestión del cuidado holístico de alta especialidad, con } \\
\text { un gran nivel de competencia en su desempeño } \\
\text { profesional a través de la aplicación de la ciencia de } \\
\text { enfermería. Formar especialistas altamente } \\
\text { calificados, para la ejecución de intervención de } \\
\text { enfermería a pacientes en estado crítico que } \\
\text { garanticen un cuidado holístico }\end{array}$ \\
\hline Chile & $\begin{array}{l}\text { Especialización en } \\
\text { Cuidados Críticos del } \\
\text { Adulto. }\end{array}$ & $\begin{array}{l}\text { Universidad } \\
\text { Católica del } \\
\text { Maule }\end{array}$ & 2 semestres & $\begin{array}{l}\text { Fortaceler competencias teórico-clínicas en la } \\
\text { entrega de cuidados de enfermería fundamentados, } \\
\text { oportunos y seguros, otorgados al paciente } \\
\text { críticamente enfermo }\end{array}$ \\
\hline
\end{tabular}

Fuente: Elaboración propia, Página Web de cada Universidad 
Esta tabla permite evidenciar que a nivel latinoamericano en tiempo de duración de los programas de especialidad fluctúa entre 1 a 2 años, y que estas especialidades concentran su proceso de formación en personas adultas. Este filtro se realizó específicamente para determinar las oportunidades de formación a nivel latinoamericano en el cuidado crítico de adultos, por ser el grupo etareo mas afectado por la pandemia.

Según el SNIES en Colombia se ofertan 26 programas de postgrado en el nivel de especialización de enfermería del total de programas. En este nivel 6 programas se ofertan en la especialidad del cuidado crítico del adulto. Al refinar la búsqueda por el campo específico de formación del programa, se evidencia que en Colombia se ofertan, en la actualidad, solamente 5 programas de especialización en Enfermeria (tabla 2), dirigidos específicamente al cuidado crítico del adulto.

Tabla 2

Oferta de programas de Especialidad en

Enfermeria en Cuidado Crítico en Colombia

\begin{tabular}{|c|c|l|c|c|c|c|}
\hline Universidad & Carácter & \multicolumn{1}{|c|}{ Nombre } & Créditos & Semestres & Admisión & Área de Influencia \\
\hline $\begin{array}{c}\text { Universidad del } \\
\text { Valle }\end{array}$ & Oficial & $\begin{array}{l}\text { Especialización en Enfermería en } \\
\text { Cuidado Crítico del Adulto }\end{array}$ & 30 & 3 & Por Cohorte & Valle del Cauca \\
\hline $\begin{array}{c}\text { Universidad del } \\
\text { Norte }\end{array}$ & Privado & $\begin{array}{l}\text { Especialización en Enfermería } \\
\text { Cuidado Critico Adulto }\end{array}$ & 60 & 2 & Anual & Atlántico \\
\hline $\begin{array}{c}\text { Fundación } \\
\text { Ciencias de la } \\
\text { Salud }\end{array}$ & Privado & $\begin{array}{l}\text { Especialización en Enfermería en } \\
\text { Cuidado Crítico del Adulto }\end{array}$ & 34 & 2 & Semestral & Bogotá D.C. \\
\hline $\begin{array}{c}\text { Universidad CES } \\
\text { Cunivado }\end{array}$ & $\begin{array}{l}\text { Especialización en Fisioterapia en } \\
\text { Cuidado Crítico del Adulto }\end{array}$ & 62 & 3 & Anual & Antioquia \\
\hline $\begin{array}{c}\text { Universidad de } \\
\text { Santander - } \\
\text { UDES }\end{array}$ & Privado & $\begin{array}{l}\text { Especialización de enfermería en } \\
\text { cuidado crítico para el adulto }\end{array}$ & 25 & 2 & Semestral & Santander \\
\hline $\begin{array}{c}\text { Universidad de } \\
\text { Antioquia }\end{array}$ & Oficial & $\begin{array}{l}\text { Especialización en Enfermería en } \\
\text { Cuidado al Adulto en Estado } \\
\text { Crítico de Salud }\end{array}$ & 36 & 3 & Semestral & Antioquia \\
\hline
\end{tabular}

Fuente: Elaboración propia, pagina Web de las Universidades

Esta tabla muestra que los programas de formación tienen una duración de 2 a 3 semestres y la oferta se concentra en las grandes capitales colombianas, dejando sin cobertura el centro sur del país, área que corresponde al departamento del Tolima.

\subsection{Disponibilidad de profesionales cualificados para atender la pandemia}

Si bien en Colombia no se ha establecido por ley o formalmente una razón enfermero/paciente para los servicios de las instituciones hospitalarias, en el sistema de salud colombiano desde 1993, se han venido implementando estrategias de reducción de costos a partir de la disminución de personal profesional de enfermería, con un aumento del número de pacientes por enfermero profesional.

Esta tendencia ha resultado preocupante, aun para el propio Ministerio de Salud y Protección Social, (Ministerio de la Salud y Proteccion Social, 2010) que destaca cómo el diseño organizacional y las condiciones de trabajo para promover un mejor desempeño en los profesionales de la salud se constituyen en dos de los elementos centrales cuando de la búsqueda de la seguridad del paciente se trata. Refiriéndose particularmente a enfermería se ha pronunciado el Consejo Técnico Nacional de Enfermería (Consejo Técnico Nacional de Enfermería, 2019), que alerta sobre la falta de estándares para regular la proporción de profesionales por paciente, área de 
desempeño y complejidad de los servicios y pacientes para garantizar la calidad de la atención que se brinda, así como sobre la sustitución por personal auxiliar, distorsionándose su perfil ocupacional

En un estudio realizado por (Garcia \& Togeiro, 2012), sobre la relación de la asignación de personal de enfermería con indicadores de resultado de la calidad de la atención en unidades de cuidados intensivos de adultos se llegaron entre otras a las siguientes conclusiones: sobrecarga del trabajo asignado a los profesionales de enfermería en las unidades de cuidado intensivo, existe una correlación entre el numero de profesionales y la ocurrencia de eventos adversos de manera inversa, es decir a menor número de profesionales mayor incidencia de mortalidad, infecciones nosocomiales y extubaciones accidentales.

Por otra parte, como lo han señalado varios teóricos de la calidad, la experiencia de los profesionales es un factor que afecta a los procesos y los resultados. Aun así, en este estudio se encontró un importante porcentaje de profesionales jóvenes y con poca experiencia trabajando en las unidades de cuidado intensivo. Es necesario en este sentido, trabajar en políticas de talento humano que promuevan la transferencia de conocimientos de los expertos hacia los novatos. Se requiere incentivar la formación posgraduada en cuidado crítico y promover que el profesional sea ubicado en el servicio para el cual se ha preparado a nivel de posgrado. Habrá que trabajar en una cultura organizacional que reconozca, desde el nivel estratégico, que tener formación posgraduada en enfermería aporta a la organización

Aunado a esta necesidad de formación del profesional de Enfermería es apremiante tener en cuenta el último Ranking de la OCDE donde se ubica a Colombia en los últimos puestos en número de médicos y enfermeros. En cuanto al número de médicos, nuestro país se encuentra ocupando uno de los peores lugares en el ranking, con 1,8 médicos por cada 1.000 personas. Sin embargo, peor posición dejan las cifras en relación con el personal de enfermería, ubicando a Colombia en el último lugar, con un índice de 1,1 por cada 1.000. También según este Ranking, Colombia se ubica en el último lugar en la proporción entre el personal de medicina y el de enfermería, siendo esta apenas de 0,6 por ciento. Brasil y Colombia son, pues, los únicos dos países de los clasificados por la OCDE en este informe con menos personal de enfermería que de medicina.

Es pertinente destacar que los recursos humanos son la base del sistema de salud y son el pilar fundamental del ejercicio de las prácticas sanitarias que incluyen la planificación de la oferta y la demanda de recursos humanos de salud es un problema importante para la mayoría de los países. La escasez de recursos humanos, el subempleo y el desempleo, los desequilibrios de la combinación de capacidades y la mala distribución geográfica son algunos de los problemas más graves que se plantean en el plano nacional y dentro de las organizaciones. La situación de la necesidad de Enfermeras especialistas es aún más complicada, debido a que el no contar con personas con formación posgraduada se ve reflejada en problemas como:

- $\quad$ Demanda especializada de cuidados no atendida;

- $\quad$ Acceso desigual a los cuidados;

- $\quad$ Desequilibrios geográficos, laborales, institucionales y en las especialidades.

- $\quad$ Calificaciones excesivas o insuficientes de los recursos humanos;

Desde el punto de vista de las condiciones laborales del talento humano se encuentra:

- Numerosas bajas en los recursos humanos.

- Desempleo o subempleo; y

- $\quad$ Retraso en la respuesta para adaptarse a las tendencias de la atención de salud (es decir, tecnología y procedimientos nuevos, etc.).

Colombia no es ajena a esta problemática, enfrentando una situación similar a la del resto de Europa y Centroamérica en relación con la escasez de enfermeras, debido a la gran migración de profesionales, aunada a esto la falta de acceso a estudios de postgrado, está causando efectos adversos para la salud y el bienestar de 
las poblaciones que requieren cuidados de salud de calidad y más en estos momentos de pandemia. Situaciones estas que motivan a las Instituciones formadoras a plantear alternativas que inviten a los profesionales a permanecer en la región, a través del ofrecimiento de programas de formación especializada, accesibles tanto económicamente, como por ubicación geográfica. Es necesario que las Instituciones de Salud reconozcan la interacción positiva que existe entre las políticas que direccionan la formación en salud y el ordenamiento del mercado laboral de los recursos humanos en salud como un aspecto fundamental y básico para el buen funcionamiento del sistema de salud y de la prestación de servicios de calidad.

Para las Instituciones prestadoras de Servicios de Salud, la preocupación con relación a la dotación adecuada del personal debe referirse no solo al número y la calidad de personas necesarias para dispensar los cuidados a los pacientes, especialmente en servicios como el cuidado crítico se deben tener en cuenta variables como la complejidad de los pacientes, el nivel de formación del personal y su distribución.

Nuestro país ha venido enfrentando transformaciones sociales, culturales y económicas relacionadas con fenómenos que afectan la población mundial como el sedentarismo, los altos niveles de estrés, el desempleo, que hacen parte de nuestra realidad nacional y regional como la violencia, el desarraigo y desplazamiento forzado, la malnutrición; que han determinado un perfil epidemiológico dinámico y cambiante, que se ve reflejado en las transformaciones en los estilos de vida, en las condiciones de salud de la población, favoreciendo la aparición de procesos patológicos crónico- degenerativos y enfermedades agudas relacionadas con nuevas formas de vida.

Estas nuevas formas de enfermar y morir, requieren de un personal de salud altamente entrenado para dar respuesta oportuna y de calidad a las necesidades de la población. Le compete a las Instituciones formadoras garantizar que proveerán a las regiones del personal competente según el área de desempeño.

Para el caso de enfermería, los adelantos científico-técnicos, la cualificación de las Instituciones de salud, los requerimientos de cuidados de enfermería humanizados en medio de las Unidades altamente tecnificadas, ha permitido que se genere la necesidad de desarrollar programas de formación que respondan a las necesidades de salud de las personas y las familias que se enfrentan a una situación crítica de salud.

\subsection{Contexto de número de camas disponibles para la atención de pacientes con Covid en el Tolima y los profesionales cualificados disponibles}

Para el Departamento del Tolima en los últimos doce años, dentro de las cuatro primeras causas de mortalidad general se encontraron: En primer lugar, las relacionadas con enfermedades del sistema circulatorio, especialmente las enfermedades isquémicas del corazón, con una tendencia de 133,2 por cada 100.000 habitantes; las enfermedades cerebrovasculares con un comportamiento fluctuante, tendiente al aumento; y las enfermedades hipertensivas con una tendencia de 22,0 por cada 100.000 habitantes (SIVIGILA, 2017). De igual manera, en las causas externas de mortalidad se encontraron aquellas relacionadas con agresiones (Homicidios), con un comportamiento oscilante tendiente al aumento; los accidentes de transporte terrestre presentaron un aumento, siendo de 21,1 por cada 100.000 habitantes y; las lesiones autoinflingidas intencionalmente (suicidios) también hacen parte de este listado, constituyéndose todas ellas en situaciones que ponen en riesgo la vida y requieren manejo especializado en Unidades de Cuidado Intensivo.

Teniendo en cuenta las principales causas de mortalidad para el departamento del Tolima, se evidencia la necesidad de que la atención especializada de enfermedades tan severas requieran para su manejo de personal bien instruido, de tal manera, que exista un manejo integral no solamente de estas patologías, sino también de los programas de salud dirigidos a la población del departamento. 
Asimismo, al analizar la situación actual de las Unidades de Cuidado Intensivo en el departamento del Tolima, se evidencia que cuenta con 17 IPS hospitalarias habilitadas para la prestación de los servicios de Cuidado Intensivo e Intermedio Adulto. Estas 17 IPS se encuentran ubicadas en los municipios de Ibagué, Líbano, Espinal, Chaparral y Mariquita. El total de camas para el manejo del paciente críticamente enfermo en el Tolima son 292 (Fajardo, Figueroa, \& Nuñez, 2020). El manejo de estos pacientes ubicados en los servicios de Cuidado Intensivo e Intermedio Adulto, requieren la presencialidad de un Médico Especialista en Medicina Crítica y Cuidado Intensivo por cada 10 pacientes en estas unidades por turno y una enfermera especialista por cada 5 pacientes hospitalizados

El déficit de enfermeras intensivistas en el departamento se calcula en más de 120, dadas las condiciones del trabajo por turnos, los días de descanso, y los imprevistos propios de la administración de talento humano; estos cálculos se traducen en la necesidad de enfermeras intensivistas en nuestra región. A la fecha en el municipio de Ibague solo cuentan con el titulo de Especialista en Cuidado Critico 13 enfermeras, igual forma, el hacinamiento generalizado presente en todas las clínicas del país, la baja cobertura en atención de este tipo, y las proyecciones para el departamento del Tolima con los establecimientos de nuevas clínicas y por ende la disponibilidad de un mayor número de camas hospitalarias y de cuidado crítico, demuestra el desbalance existente entre oferta y demanda de la especialidad de Medicina Critica y Cuidado Critico en la región.

\section{Conclusiones}

La Universidad del Tolima ha cumplido un rol determinante durante la pandemia por COVID-19, teniendo en cuenta los retos impuestos por esta situación, planeó e incrementó su oferta académica, organizó sus procesos de formación, investigativos, de proyección social y administrativos con el fin de poder responder con altos estándares de calidad impuestos para las instituciones de Educación Superior.

La pandemia por COVID-19 ha traído a la Universidad del Tolima, como única institución de educación superior pública del Departamento, líder en la formación de talento humano en salud, múltiples oportunidades de intervención; permitiéndole desarrollar aún más su potencial y liderazgo en la formación de talento humano en salud de la región.

El proveer a la ciudad de Ibagué y al Departamento del Tolima el talento humano suficiente y cualificado para la utilización correcta de las herramientas disponibles y la implementación de los cuidados requeridos segundo a segundo, han constituido durante la pandemia por COVID-19, una prioridad para la Universidad del Tolima.

La importancia de reconocer como en el personal calificado de enfermeria en cuidado crítico debe contar tambien con las condiciones laborales durante y pos pandemia con el fin de garantizar la salud de todos los actores presentes en la primera linea de atención.

\section{Referencias bibliográficas}

Chica, C., Peña, L., Villamarin, H., Jorge, M., Rodriguez, L., Lozano, W., \& Vargas, M. (2020). Cuidado respiratorio y COVID. Acta Colombiana de Cuidado intensivo, 20(1), 108-117.

Congreso de la República de Colombia. (1992). Ley 30 de 1992. Recuperado el 16 de Julio de 2020, de Consejo Nacional de Acreditación Colombia: https://www.cna.gov.co/1741/articles-186370_ley_3092.pdf

Consejo Técnico Nacional de Enfermería. (2019). Plan Nacional de Enfermería 2020-2030. (en construccion). Santa Fé de Bogotá. Recuperado el 21 de Marzo de 2020, de https://www.oceinfo.org.co/difusion/material-en-general/send/4-normatividad/147-propuesta-plannacional-de-enfermeria-2020-2030 
Fajardo, E., Figueroa, R., \& Nuñez, M. (2020). Análisis de la formación de especialistas en Medicina Crítica en la Ciudad de Ibagué. Revista Edu-Fisica.com, 119-130.

Garcia, C., \& Togeiro, F. (2012). Tiempo de atención de Enfermeria en la Unidad de Cuidados Intensivos. Revista Latinoamericana de Enfermería, 20(4), 42-51.

Gómez, V., \& Celis, J. (2009). Sistema de aseguramiento de la calidad de la educación superior: consideraciones sobre la acreditación en Colombia. Revista Colombiana de Sociologia, 32(2), 87-110.

Gonzalez, C. (2015). Competencias profesionales en enfermeras que desempeñan su labor en Cuidados Intensivos. ALADEFE. Revista Iberoamericana de Investigación y Educación en Enfermería, 5(1), 35-46.

Grant, Maria J., and Andrew Booth. 2009. "A Typology of Reviews: An Analysis of 14 Review Types and Associated Methodologies." Health Information and Libraries Journal 26(2):91-108.

Khangura, Sara, Kristin Konnyu, Rob Cushman, Jeremy Grimshaw, and David Moher. 2012. "Evidence Summaries: The Evolution of a Rapid Review Approach." Systematic Reviews 1(1):1-9.

Matiz, H. (2016). Historia del Cuidado Intensivo en Colombia. Revista Colombiana de Anestesiología, 44(3), 190193.

Ministerio de la Salud y Proteccion Social. (2010). Recuperado el 26 de Septiembre de 2020, de https://www.minsalud.gov.co/sites/rid/Lists/BibliotecaDigital/RIDE/DE/CA/Guia-buenas-practicasseguridad-paciente.pdf

Ministerio de Salud y Protección Social. (2016). Ministerio de Salud. gov.co. Recuperado el 28 de Septiembre de 2020, de https://www.minsalud.gov.co/sites/rid/Lists/BibliotecaDigital/RIDE/DE/DIJ/decreto-780-unicomodificado-2016.pdf

SIVIGILA, S. d. (2017). ANALISIS DE SITUACION DE SALUD - ASIS. Departamento del Tolima.

Tricco, Andrea C., Jesmin Antony, Wasifa Zarin, Lisa Strifler, Marco Ghassemi, John Ivory, Laure Perrier, Brian Hutton, David Moher, and Sharon E. Straus. 2015. "A Scoping Review of Rapid Review Methods." BMC Medicine 13(1).

Universidad del Tolima. (2018). Documentos Administrativos Universidad del Tolima. Recuperado el 25 de Agosto de 2020, de http://administrativos.ut.edu.co/images/VICEACADEMICA/Autoevaluacion/Informe_condiciones_iniciales. pdf

Esta obra está bajo una Licencia Creative Commons Attribución-NoCommercial 4.0 International

(cc) BY-NC 